\title{
BANCO DE IMAGENS MAMOGRÁFICAS PARA TREINAMENTO NA INTERPRETAÇÃO DE IMAGENS DIGITAIS*
}

\author{
Silvio Ricardo Pires ${ }^{1}$, Regina Bitelli Medeiros ${ }^{2}$, Homero Schiabel ${ }^{3}$
}

Resumo OBJETIVO: Disponibilizar aos profissionais da radiologia um "software" para treinamento na interpretação de imagens mamográficas em sistemas digitais. MATERIAIS E MÉTODOS: Foi desenvolvido um "software" em Delphi associado a uma base de dados em Interbase, com a finalidade de armazenar imagens de exames mamográficos associados aos seus laudos em categorias BI-RADS ${ }^{\circledR}$. As imagens foram previamente qualificadas e digitalizadas em "scanner" a laser Lumiscan 75. 0 treinamento se faz com imagens apresentadas em monitores comerciais de 17 polegadas, no tamanho $18 \times 24 \mathrm{~cm}$. 0 "software" permite visualizar cada projeção das mamas individualmente, médio-lateral oblíqua e crânio-caudal, ou as quatro imagens simultaneamente. Permite acessar as imagens e os laudos existentes ou interpretar as imagens utilizando o sistema de categorias BI-RADS ${ }^{\circledR}$, em que o "software" compara o laudo do usuário com as informações do banco de dados, apontando acertos e erros da interpretação. RESULTADOS: 0 usuário adquire familiaridade com sistemas digitais, laudos em categorias BI-RADS ${ }^{\circledR}$ e aspectos de qualidade do processo gerador das imagens relativo à detecção de fibras e microcalcificações. CONCLUSÃO: 0 "software" disponível na intranet da Universidade Federal de São Paulo é ferramenta valiosa para os profissionais interessados em sistemas digitais. Unitermos: Banco de dados; Mamografia; Digital; Controle de qualidade.

Abstract Digital image database for mammography interpretation training.

OBJECTIVE: To make a software available for training professionals in the interpretation of mammography images on digital systems. MATERIALS AND METHODS: The software was developed in Delphi and linked to a Interbase database to store mammographic images in association with the BI-RADS ${ }^{\circledR}$ category results. Images were previously qualified and digitized using a Lumiscan 75 laser scanner. Training is done on $18 \times$ $24 \mathrm{~cm}$ images displayed on a 17" commercial monitor. The software allows displaying of each breast view individually, medial-lateral oblique and cranial-caudal views, or four images simultaneously. The user selects an exam from the database and makes a diagnosis classifying the image into BI-RADS ${ }^{\circledR}$ categories. At the end of the process, the software compares the user's interpretation with the data stored in the database and than yields scores. RESULTS: The user becomes familiar with digital systems, images interpretation using BI-RADS ${ }^{\circledR}$ categories and with the quality of the image generating process regarding the detection of fibers and microcalcifications. CONCLUSION: The software is available at "Universidade Federal de São Paulo", São Paulo, Brazil, intranet and is a valuable tool for training professionals interested in digital systems.

Key words: Data base; Mammography; Digital; Quality control.

\section{INTRODUÇÃO}

A mamografia é considerada o método mais eficiente de rastreamento do câncer de mama através da busca de estruturas que

* Trabalho realizado na Coordenadoria de Física e Higiene das Radiações (CFHR) - Departamento de Diagnóstico por Imagem (DDI) da Universidade Federal de São Paulo/Escola Paulista de Medicina (Unifesp/EPM), São Paulo, SP.

1. Bacharel em Física pela Universidade de São Paulo (USP), CFHR - DDI-Unifesp/EPM.

2. Professora Doutora, Chefe da Coordenadoria de Física Médica da Unifesp/EPM.

3. Professor Doutor de Engenharia Biomédica, Departamento de Engenharia Elétrica da Escola de Engenharia de São Carlos da Universidade de São Paulo (EESC-USP).

Endereço para correspondência: Silvio Ricardo Pires. Coordenadoria de Física e Higiene das Radiações, Universidade Federal de São Paulo. Rua Botucatu, 659, Vila Clementino. São Paulo, SP, 04023-900. E-mail: silvio@cfhr.epm.br.

Recebido para publicação em 3/9/2003. Aceito, após revisão, em 3/10/2003 possam indicar a presença de anormalidades ou tumores ${ }^{(\mathbf{1 , 2})}$. Dentre essas estruturas podemos destacar as microcalcificações, que freqüentemente estão associadas a tumores de mama ${ }^{(2-6)}$. No entanto, devido ao tamanho reduzido dessas estruturas (da ordem de 0,5 mm de diâmetro), sua visualização é muito difícil e exige grande experiência do especialista. Mesmo assim, muitas vezes são passadas despercebidas, visto que o processo gerador das imagens pode apresentar várias limitações que prejudicam a sua nitidez, como ruídos aleatórios, variações nas condições de processamento, ponto focal com dimensões inadequadas, presença de artefatos, etc. ${ }^{(2)}$.

Os programas de rastreamento têm por objetivo a detecção precoce do câncer de mama em mulheres assintomáticas e geralmente são subsidiados por órgão governamental $^{(8)}$. Vários países que fizeram o rastreamento provaram que a taxa de detecção tem sido superior ao risco associado à exposição à radiação. Já foi provado, em estudos de países como Suécia, Inglaterra e Escócia, que o rastreamento mamográfico apresenta benefícios maiores do que os riscos de indução do câncer, havendo um ganho de anos de vida para as mulheres que foram rastreadas. O risco é teoricamente maior para mulheres mais jovens ou com mamas densas e principalmente para aquelas que têm histórico familiar de câncer de mama e podem ser mais suscetíveis aos efeitos da radiação. Kopans estimou este risco em torno de 1 para $1.000 .000^{(\mathbf{9 , 1 0})}$. 
A mamografia para fins de diagnóstico é aquela realizada nas pacientes com sintomas específicos, tais como nódulo mamário, fluxo papilar ou alteração do exame físico (espessamento mamário), que geralmente é indicada e acompanhada pelo ginecologista ou mastologista. Também se incluem nesse grupo os exames das pacientes de alto risco (mesmo os periódicos) e os controles pós-operatórios ${ }^{(\mathbf{8})}$. Nesta categoria de procedimento não se discute a justificativa do método, porém, tal como no caso do rastreamento, torna-se relevante garantir a qualidade do processo em benefício direto das pacientes.

Deve-se notar que vários fatores que influenciam na qualidade da imagem devem ser respeitados, para que o rastreamento seja bem sucedido ${ }^{(\mathbf{1 0 , 1 1})}$. A taxa estimada de exames falso-negativos é de aproximadamente $5 \%$ a $15 \%$ dos casos. A falha na detecção para a caracterização do câncer pode também ser atribuída a fatores técnicos ou a limitações operacionais ${ }^{(\mathbf{1 0})}$.

Paralelamente à qualidade do processo gerador da imagem, a precisão do diagnóstico é diretamente dependente da subjetividade na interpretação das imagens radiológicas. O principal fator que leva a um mesmo observador possuir mais de uma interpretação de uma mesma imagem normalmente está relacionado ao cansaço físico e ao esforço após algumas horas de interpretação ou leitura radiológica. Outro fator de relevância na interpretação de imagens mamográficas está na experiência do observador. O treinamento clínico neste tipo de modalidade de imagem é crucial para a detecção precoce de tumores malignos e redução dos falso-positivos ${ }^{(11,12)}$.

Uma característica muito importante e que pode contribuir de forma intrínseca à subjetividade na interpretação da imagem é o grau de enegrecimento desta. Este parâmetro está intimamente relacionado com as condições de visibilidade disponíveis à interpretação, às características pessoais e ao grau de treinamento do especialista. $\mathrm{O}$ alto grau de enegrecimento exige o uso de negatoscópios especiais com alta luminância, nem sempre disponíveis ao uso, sob pena de se reduzir a detectabilidade de estruturas suspeitas ${ }^{(13)}$.

Condições adequadas para a interpretação da imagem mamográfica são essen- ciais para a detecção de pequenas estruturas como as microcalcificações. Negatoscópios de luminância igual ou superior a $3.000 \mathrm{~cd} / \mathrm{m}^{2}$, máscara para limitação da luz refletida sobre áreas do filme, ambiente com baixa luminosidade, concentração na leitura do exame, são itens que, associados à experiência em achados clínicos e à capacidade de correlacionar resultados, contribuíram para a redução dos falsos positivos e negativos ${ }^{(11,13,14)}$

As limitações do sistema tela-filme são um dos fatores mais significativos na redução da visualização de tumores de mama e microcalcificações, principalmente em pacientes com tecido fibroglandular denso, para o qual a perda de detecção do câncer é mais freqüente.

O correto posicionamento, que permitirá que todo o tecido da mama seja radiografado, e a compressão adequada, que reduzirá a espessura uniformizando os tecidos da mama e o espalhamento, são componentes fundamentais para a melhoria do contraste da imagem. Imagens de alto contraste são muitas vezes impostas como padrão clínico, a fim de assegurar a detectabilidade de estruturas diminutas e identificar estruturas suspeitas de malignidade que possuam coeficientes de absorção de raios $\mathrm{X}$ bastantes similares ao do tecido normal.

Segundo recentes estatísticas divulgadas pelo Instituto Nacional do Câncer (INCA), no Brasil uma em cada 12 mulheres poderá desenvolver câncer de mama. $\mathrm{O}$ mais alarmante é que, entre os tipos de câncer, este é o que mais mata as brasileiras. O que também deve ser levado em consideração, neste caso, é o impacto psicológico causado pela doença, pois envolve fatores como a sexualidade e a autoestima $^{(\mathbf{1 5}, \mathbf{1 6})}$. Tais pesquisas também apontam que, no Brasil, 75\% dos casos são diagnosticados quando a doença já se encontra em estágio avançado, o que dificulta muito o tratamento ${ }^{(\mathbf{1 7})}$

Na tentativa de uniformizar a linguagem médica utilizada nos laudos mamográficos, o Colégio Americano de Radiologia desenvolveu um sistema especial que expressa os achados mamográficos em categorias, denominado Breast Imaging Reporting and Data System (BI-RADS ${ }^{\circledR}$ ). Trata-se do mais completo sistema já elaborado para garantir a padronização do laudo mamográfico, que propõe condutas clínicas conforme a categorização. Seu objetivo principal é diminuir as diferenças de conduta inerentes à variabilidade (ou discordância) dos observadores na interpretação da imagem mamográfica, facilitando também a obtenção do resultado final do exame (18-20). $^{\text {. }}$ Esta classificação é dividida em:

\section{a) Avaliação incompleta}

Categoria 0: Avaliação adicional de imagem é necessária. Inclui compressão localizada, ampliação, incidências adicionais, ultra-sonografia. Esta categoria é freqüentemente utilizada em exames de rastreamento, mas raramente quando se considera o estudo imaginológico completo.

\section{b) Avaliação completa}

Categoria 1: Negativa (cerca de 0\%). As mamas são simétricas, não há nódulos, distorções de arquitetura ou calcificações suspeitas.

Categoria 2: Achados benignos (cerca de $0 \%$ ). São achados que merecem descrição, porém tipicamente benignos: fibroadenomas calcificados, calcificações secretórias (benignas), lesões com conteúdo de gordura (cisto oleoso, lipoma), lesões com densidades mistas e implantes.

Categoria 3: Achados provavelmente benignos (até $2 \%$ ). Pode-se pedir um controle em intervalo inferior a um ano para avaliar a estabilidade da lesão (são achados dos quais não se espera alterações, pois são considerados benignos, porém é preferível avaliação precoce). Por exemplo: fibroadenoma não-calcificado.

Categoria 4: Achados suspeitos - considerar biópsia (de $2 \%$ a $90 \%$ ). Lesões que não têm características de câncer de mama, mas também não podem ser classificadas como provavelmente benignas. $\mathrm{O}$ médico e seu paciente podem tomar a decisão sobre a realização do procedimento.

Categoria 5: Achados altamente sugestivos de malignidade - prosseguir com biópsia (> 90\%)

A leitura de imagens digitais está se tornando uma realidade nos centros radiológicos, e o treinamento na leitura dessas imagens pode colaborar para a melhoria da detectabilidade, com redução dos índices de falsos positivos e negativos no diagnóstico por imagem. Com este estudo preten- 
de-se disponibilizar à área médica uma ferramenta que permita facilitar o processo de transição dos métodos convencionais para os digitais.

\section{Objetivos}

Disponibilizar um "software" que permita o treinamento dos profissionais interessados em iniciar na área de interpretação de exames em sistemas digitais.

Propiciar o treinamento em laudos de sistemas BI-RADS ${ }^{\circledR}$ em monitores comerciais de 17 polegadas ou superiores.

\section{MATERIAIS E MÉTODOS}

A proposta deste trabalho consistiu no desenvolvimento de um "software" em Delphi 6 associado a um banco de dados Interbase 6 (ambos da Borland). O desenvolvimento deste sistema tem como base o armazenamento de imagens de exames mamográficos associados aos seus laudos em sistemas BI-RADS ${ }^{\circledR}$. O “software" consiste em um formulário em que serão representadas as imagens dos exames mamográficos, juntamente com o seu laudo, representados pelas Figuras 1 e 2 .

A Figura 1 exibe as quatro imagens mamográficas, enquanto na Figura 2 foi feita ampliação em uma das imagens para melhor visualização das estruturas, além da

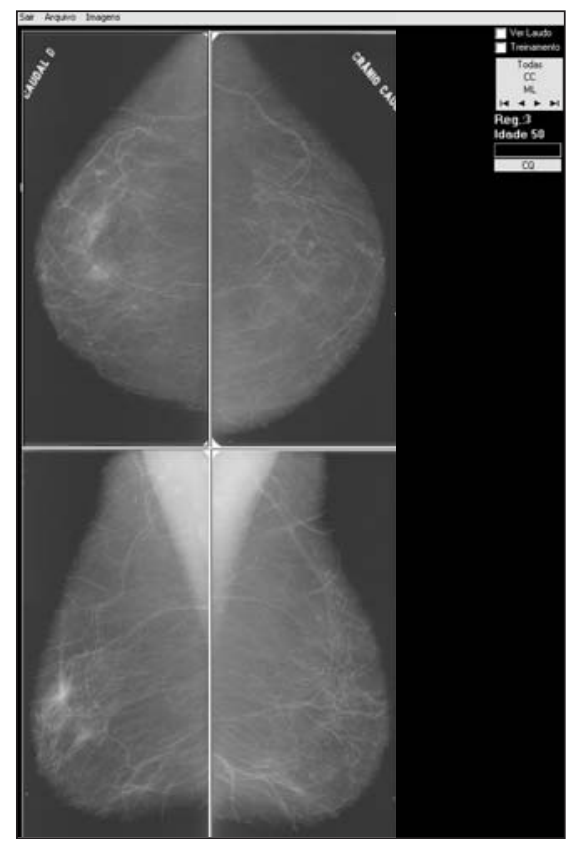

Figura 1. Tela que mostra as quatro imagens mamográficas de um exame.

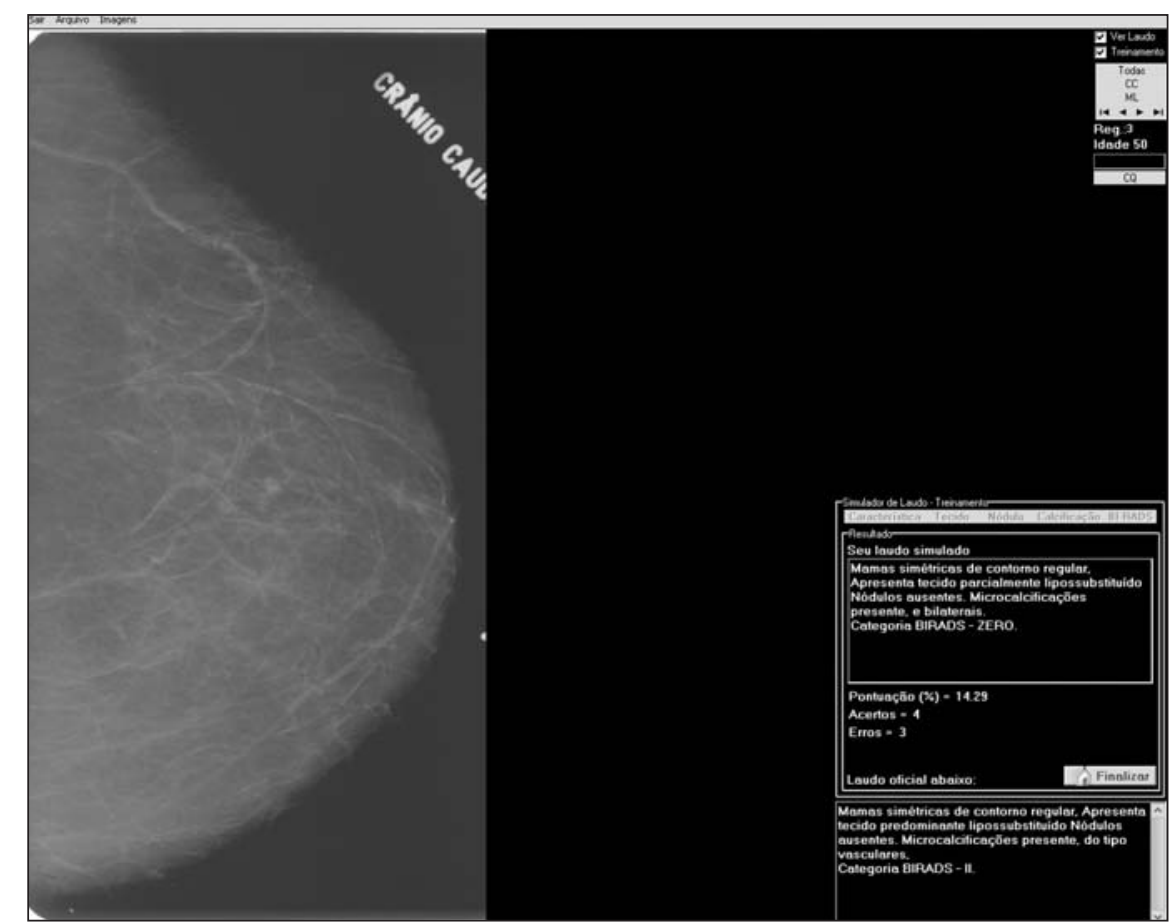

Figura 2. Tela que mostra uma imagem individual, juntamente com o sistema de avaliação do desempenho e o laudo da imagem em sistema BI-RADS ${ }^{\circledR}$.

visualização do laudo em sistema de categorias BI-RADS ${ }^{\circledR}$.

Como as imagens mamográficas exigem alta resolução, devido à sua complexa estrutura de detalhes, o "software" foi desenvolvido para trabalhar com monitores de 17 polegadas ou superiores, com resolução mínima de $1.280 \times 1.024$ pixels e com contraste mínimo de 8 bits por pixel em tons de cinza. Todas as imagens foram digitalizadas em um "scanner" a laser Lumiscan 75 (Lumisys, Inc.), com contraste de 8 bits, tamanho de pixel $(87 \pm 2) \mathrm{mm}$, em formato TIFF, e em seguida convertidas para o formato BMP, ajustando o tamanho de seu pixel para ampliação na ordem de $(240 \pm 8) \mathrm{mm}$. As imagens foram redimensionadas para o mesmo tamanho do filme mamográfico $(18 \times 24 \mathrm{~cm})$, sendo que foi feito um corte na região da imagem que não continha nenhuma informação. Finalmente, as imagens foram armazenadas no banco de dados, juntamente com o seu laudo em sistemas BI-RADS ${ }^{\circledR}$.

As imagens mamográficas foram geradas no mesmo período que imagens de simuladores foram utilizadas para qualificar o processo gerador de imagem como parte do programa de garantia de qualidade.
Essas imagens mamográficas estão, portanto, associadas a um banco de informações que disponibiliza dados sobre parâmetros técnicos relevantes à sua qualificação. Este banco de dados do programa de garantia de qualidade permite ao usuário conhecer a possibilidade de identificar microcalcificações e fibras e em que dimensões.

Foi criada, no "software", uma função que permite o treinamento de profissionais da área, em que o usuário busca um exame de interesse no banco de dados e efetua o diagnóstico utilizando o mesmo padrão do sistema BI-RADS ${ }^{\circledR}$. No final do processo o "software" faz a comparação do resultado da interpretação simulada com o armazenado no banco de dados, obtendo uma pontuação ponderada de acertos. Alternativamente, se o usuário não desejar fazer um treinamento, existe uma opção em que ele pode estudar o exame já interpretado no próprio monitor, com a finalidade de se adaptar à tecnologia digital.

Em qualquer fase do processo de apresentação das imagens no monitor o usuário pode fazer uma consulta sobre o índice de qualidade da imagem, parâmetros técnico-operacionais e de controle de qualidade sob os quais estão associadas. 


\section{RESULTADOS}

As telas do "software" possibilitam o treinamento na visualização dos exames mamográficos juntamente com o seu laudo em sistemas de categorias BI-RADS ${ }^{\circledR}$, além da avaliação do desempenho comparando o laudo simulado com o armazenado no banco de dados. Durante o treinamento, é possível que o usuário visualize os parâmetros técnico-operacionais e de controle de qualidade do período em que foram geradas as imagens, bem como o índice de qualidade dessas imagens.

A Figura 3 representa a tela do "software" que mostra o grupo de imagens crânio-caudal no mesmo tamanho físico representado pelo filme mamográfico, com o objetivo de avaliar, de forma mais eficiente, suas estruturas internas. Analogamente, é possível a visualização do grupo de imagens médio-lateral.

A Figura 4 mostra a tela do "software" que permite visualizar a imagem do simulador RMI-156 e o índice de qualidade, e os parâmetros técnicos descritos a seguir:

a) Dados referentes ao equipamento mamográfico: local de instalação, modelo, fabricante.

b) Dados referentes ao processamento: temperatura do fixador/revelador/água,

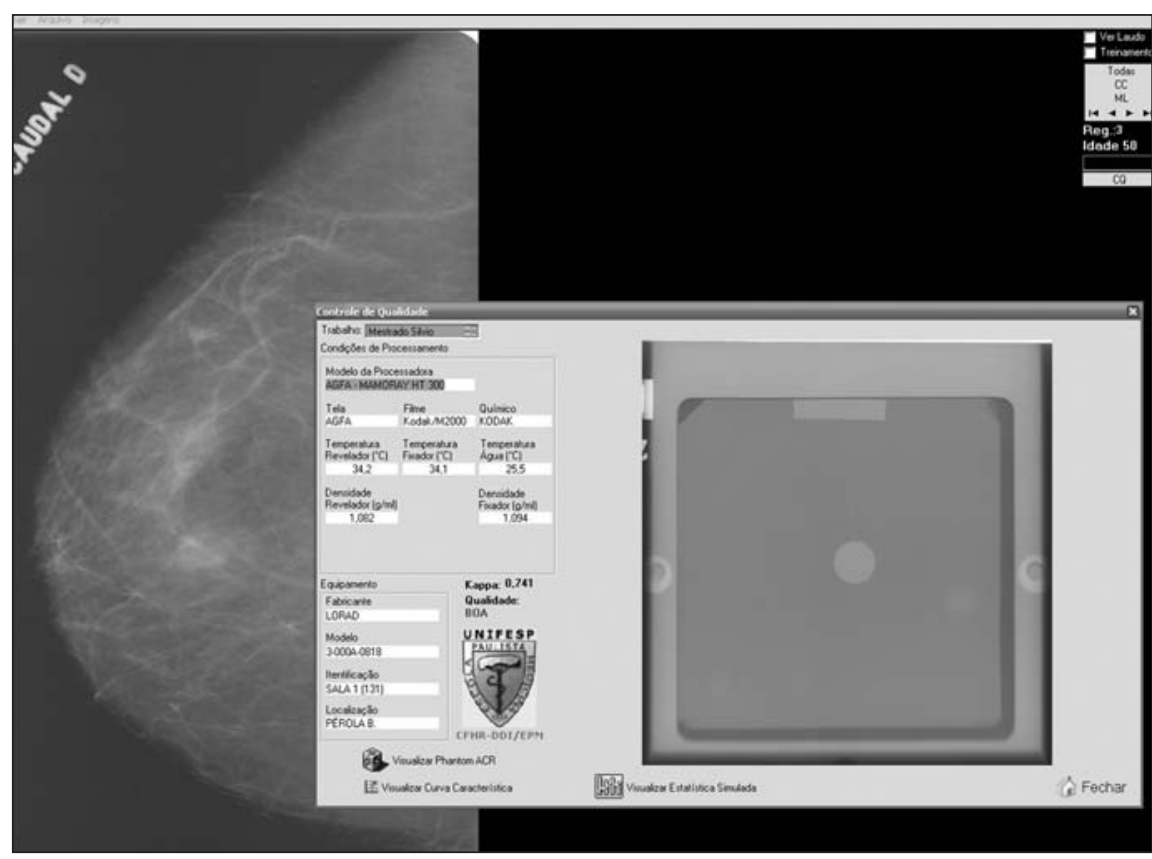

Figura 4. Tela que mostra os parâmetros de controle de qualidade que gerou esta imagem, juntamente com a imagem do simulador RMI-156. sensitometria, fabricante e modelo da processadora.

c) Dados referentes aos insumos: fabricante e modelo das telas, filmes e agentes químicos.

O objetivo de disponibilizar este tipo de informação é para que o usuário adquira

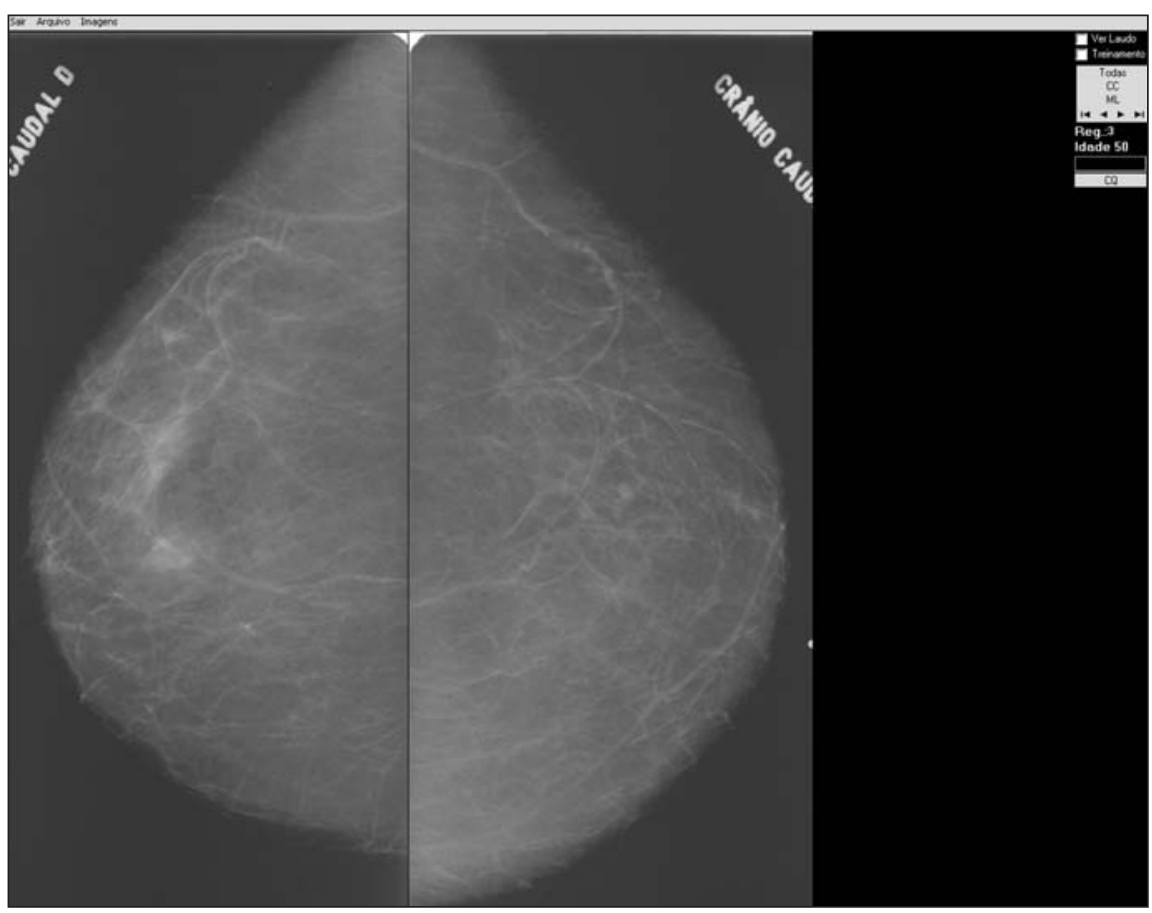

Figura 3. Tela que mostra o grupo crânio-caudal. conhecimento das condições de qualidade existentes na ocasião da realização das imagens e possa avaliar como a variação dos parâmetros físicos e técnicos pode influenciar na qualidade das imagens.

A Figura 5 representa a tela do "software" que mostra os mesmos parâmetros da Figura 4, com a diferença que aqui é apresentada graficamente a resposta sensitométrica do filme no dia em que as imagens simuladas foram geradas.

A Figura 6 representa a tela do "software" com os mesmos parâmetros da Figura 4 e inclui dois gráficos: o primeiro mostra o grau de visibilidade das estruturas em função do seu tamanho e tipo de estrutura, e o segundo gráfico apresenta sua qualificação ou nível de concordância (valor de kappa) em função do grau de exposição utilizado para a obtenção da imagem. Os valores aqui representados estão todos em função das características em que foram geradas as imagens do exame mamográfico vinculado.

\section{DISCUSSÃO}

O digitalizador Lumiscan 75 é capaz de gerar imagens no formato TIFF, com contraste de 8 ou 12 bits. Como a maioria dos monitores comerciais possui apenas uma 


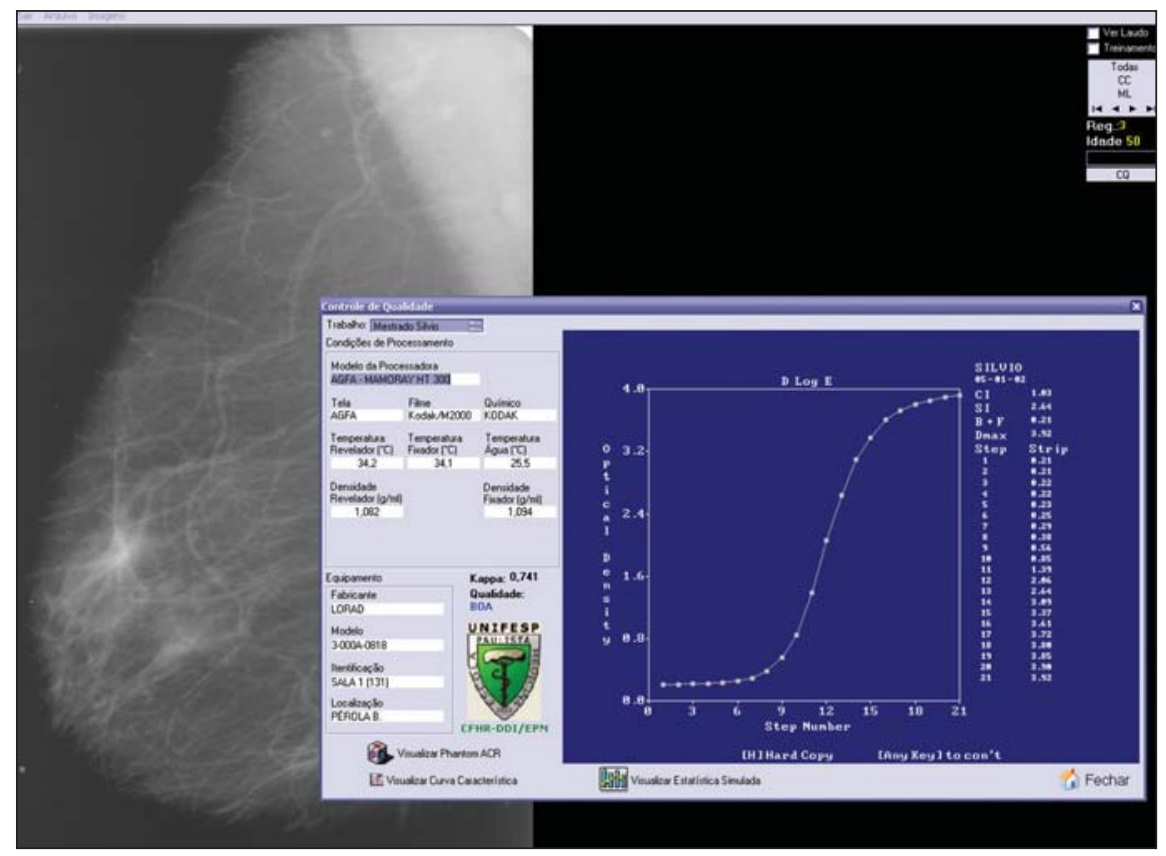

Figura 5. Tela que mostra os parâmetros de controle de qualidade e a curva característica do filme no dia em que as imagens simuladas e as imagens mamográficas foram geradas.

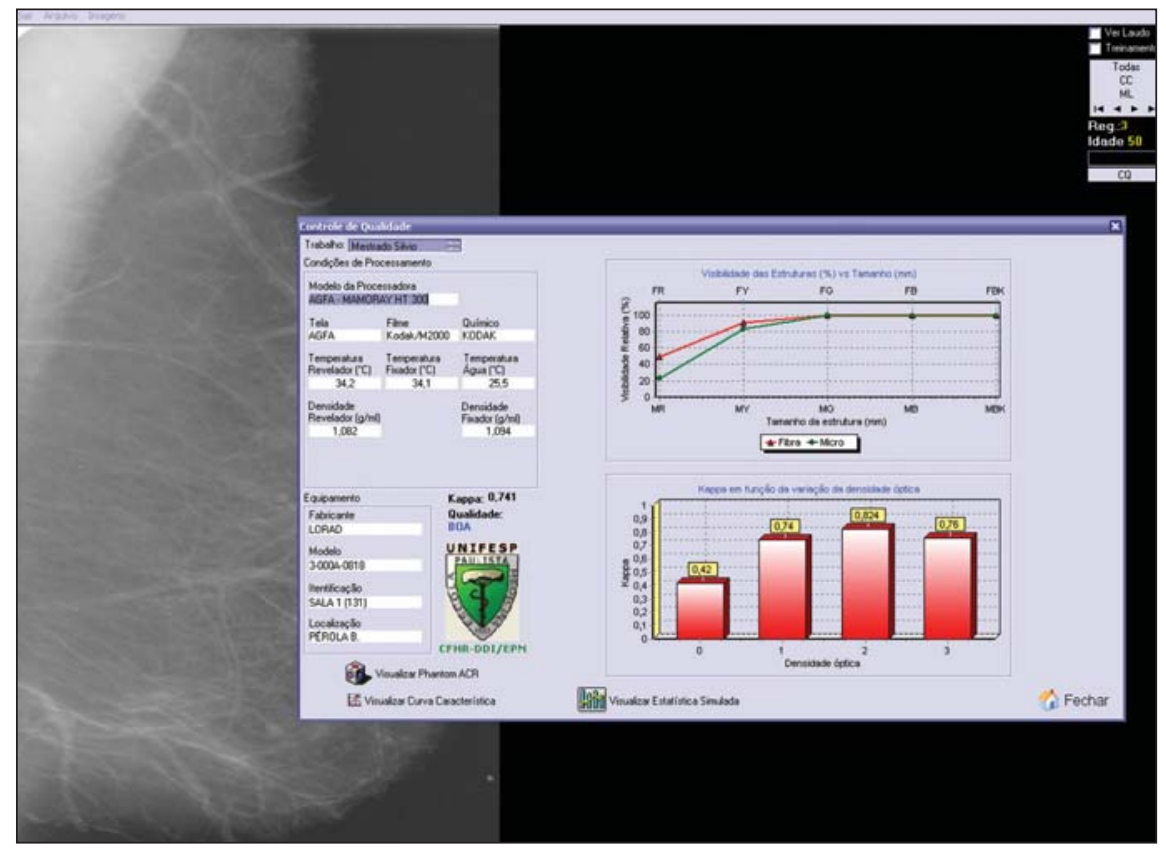

Figura 6. Tela que mostra, em função das características de formação desta imagem mamográfica, o grau de visibilidade das estruturas em função do seu tipo e tamanho, bem como o nível de concordância em relação à densidade ótica.

profundidade de cor em 8 bits, imagens de 12 bits, para serem representadas corretamente, devem sofrer um pré-processamento para serem exibidas na tela (em 8 bits). Além do que, imagens em 12 bits ocupam o dobro de espaço de armazenamento em disco, porque o sistema gera arquivos em que o poder de armazenamento de imagens dobra para o mesmo espaço em disco.

Propomos que a visualização das imagens seja feita utilizando-se monitores comerciais de 17 polegadas que possuam resolução mínima de $1.280 \times 1.024$ pixels. As imagens geradas pelo digitalizador Lumiscan 75 possuem resolução de 2.048 pixels por linha. Se apresentadas diretamente na tela do monitor comercial haveria muita dificuldade por parte do usuário, pois teria que visualizar as imagens por áreas de interesse. Por este motivo foram reduzidas de tamanho, para permitir que sejam visualizadas como um todo, como o fazem quando estão analisando o filme convencional.

O digitalizador Lumiscan 75 gera uma imagem com tamanho do pixel de $(87 \pm 1)$ $\mu \mathrm{m}$, enquanto um monitor de 17 polegadas de dimensão efetiva de $31,5 \times 24 \mathrm{~cm}$ possui tamanho de pixel de $(240 \pm 8) \mu \mathrm{m}$. Sendo assim, o menor elemento de formação da imagem do monitor é quase três vezes maior que o menor elemento da imagem digital, acarretando perda de resolução devido à perda de informação natural do sistema selecionado (monitor). Aproveitando-se desta deficiência do sistema, foi ampliado o tamanho do menor elemento de todas as imagens digitais para o mesmo tamanho do pixel do monitor, sem perda de qualidade para o observador, embora a perda de resolução persista. Uma vez que o tamanho do pixel da imagem é igual ao tamanho do pixel do monitor, a imagem encaixa-se perfeitamente na tela em tamanho real, ou seja, no mesmo tamanho do filme. Outra grande vantagem é a sensível redução do tamanho físico do arquivo que ocupa no disco rígido ${ }^{(22)}$.

Geralmente, as imagens de exames mamográficos possuem uma grande área que não contém nenhuma informação. Como a finalidade é o armazenamento em um banco de dados, para fins de otimização do tamanho dos arquivos, foram recortadas estas áreas sem informação de todas as imagens mamográficas antes de serem inseridas no banco de dados.

Sabe-se que para imagens digitais, não comprimidas, a quantidade de informação disponível para o formato TIFF e BMP(21), quando em 8 bits, é a mesma. O compilador Delphi já possui todas as rotinas internas 
para trabalhar com imagens em formato BMP. Aproveitando-se deste recurso, todas as imagens foram convertidas para este formato. Após a conversão, foram todas armazenadas no banco de dados ${ }^{(22)}$.

Benatti $^{(\mathbf{2 3})}$ desenvolveu um banco de dados com todas as imagens mamográficas digitalizadas em 12 bits, com o objetivo de, no futuro, processá-las. Entretanto, para a visualização das imagens em monitores, foi necessária a implementação de um algoritmo que convertesse as imagens para 8 bits, de forma a adequar sua representação na tela do monitor. Segundo Benatti, a conversão não gera nenhum tipo de deterioração visível na imagem, entretanto, imagens com 8 bits não são aconselháveis para processamento e sim somente para visualização no monitor.

O "software" está disponível na intranet da Universidade Federal de São Paulo (Unifesp) e será disponibilizado aos profissionais como parte integrante do programa de treinamento do Departamento de Diagnóstico por Imagem da Unifesp. Apresenta, como vantagem, baixo custo e a facilidade de acesso devido à interface amigável com o usuário.

O "software" é uma ferramenta de treinamento destinada aos profissionais médicos interessados na interpretação de imagens radiológicas digitais, que propiciará uma familiaridade com os laudos em sistema de categorias BI-RADS ${ }^{\circledR}$ e aspectos de qualidade das imagens relativos à detecção de pequenas estruturas, como fibras e microcalcificações.

\section{REFERÊNCIAS}

1. Kopans DB. Standardized mammography reporting. Radiol Clin North Am 1992;30:257-64.

2. Mitchell GW Jr, Bassett LW. Mastologia prática. $1^{\text {a }}$ ed. Rio de Janeiro: Revinter, 1988.

3. Shen L, Rangayyan RM, Desautels JEL. Detection and classification of mammographic calcifications. International Journal of Pattern Recognition and Artificial Intelligence 1993;7:1403-16.

4. Shen L, Rangayyan RM, Desautels JEL. Application of shape analysis to mammographic calcifications. IEEE Transactions on Medical Imaging 1994;13: 263-74.

5. Tao E, Ornes C, Sklansky J. Automatic detection of microcalcifications in digital mammography. Proceedings of SPIE 1998;3338(6):1450-8.

6. Canella EO. Microcalcificações. Jornal da Imagem 1999;245(1):20 caderno.

7. Nagel RH, Nishikawa RM, Papaioannou J, Doi K. Analysis of methods for reducing false positives in automated detection of clustered microcalcifications in mammograms. Med Phys 1998;25:15026.

8. Tabar L, Fagerberg CJG, Gad A, et al. Reduction in mortality from breast cancer after mass screening with mammography. Randomized trial from the Breast Cancer Screening Working Group of the Swedish National Board of Health and Welfare. Lancet 1985;i:829-32.

9. Pasqualette HA. História da mamografia, indicações e técnicas de interpretação. In: Pasqualette HA, Koch HA, Pereira PMNS, Kemp C. Mamografia atual. 1를. Rio de Janeiro: Revinter, 1998:17-29.

10. Kopans DB. La mama en imagen. $1 \underline{\underline{a}}$ ed. Madrid. Marban, 1994:14-6.

11. Christiansen CL, Wang F, Barton MB, et al. Predicting the cumulative risk of false-positive mammograms. J Natl Cancer Inst 2000;92:1657-66.

12. Beam C, Hendrick RE. Proposition: All mammo- grams should be double-read. Med Phys 1999;26: $115-8$.

13. Medeiros RB, Alves FFR, Ruberti EM, et al. Influência do brilho do negatoscópio na detectabilidade clínica de fibras e microcalcificações em imagens mamográficas. Anais do XXIX Congresso Brasileiro de Radiologia. Salvador, 2000.

14. Brooks KW, Trueblood JH, Kearfott KJ. Subjective evaluations of mammographic accreditation phantom images by three observer groups. Invest Radiol 1994;29:42-7.

15. Kemp C, Baracat FF, Rostagno R. Lesões não palpáveis da mama. Diagnóstico e tratamento. 1ํㅡ ed. Rio de Janeiro: Revinter, 2003:17-35.

16. Faulkner K, Law J. Cancers detected and induced, and associated risk and benefit, in a breast screening programme. Br J Radiol 2001;74:1121-7.

17. Escarpinati MC. Investigação de formatos e compressão de imagens digitais para processamento de imagens mamográficas de mamas densas. (Tese de Mestrado). São Carlos: Escola de Engenharia de São Carlos da Universidade de São Paulo, 2002.

18. American College of Radiology (ACR). Breast imaging reporting and data system $\left(\mathrm{BI}-\mathrm{RADS}^{\circledR}\right)$. 3rd ed. Reston: American College of Radiology, 1998.

19. I Reunião de Consenso da Radiologia - padronização dos laudos mamográficos. Colégio Brasileiro de Radiologia, Sociedade Brasileira de Mastologia e Federação das Sociedades de Ginecologia e Obstetrícia, 1998.

20. Kemp C, Martinelli SE. Sistemas BI-RADS ${ }^{\circledR}$. In: Kemp C, Baracat FF, Rostagno R. Lesões não palpáveis da mama. Diagnóstico e tratamento. $1^{\mathrm{a}} \mathrm{e}$ ed. Rio de Janeiro: Revinter, 2003:17-35

21. Adobe Developers Association. TIFF: Revision 6.0 technical specifications. June 1992.

22. Pires SR, Medeiros RB, Schiabel H, Elias S. Qualificação da imagem mamográfica simulada. Anais do XVIII Congresso Brasileiro de Engenharia Biomédica 2002;2:51-4.

23. Benatti RH. Elaboração de um banco de imagens mamográficas digitalizadas. (Tese de Mestrado). São Carlos: Escola de Engenharia de São Carlos da Universidade de São Paulo, 2003. 